\title{
Phytochemicals and Antioxidant Capacities from Dacryodes rostrata Fruits
}

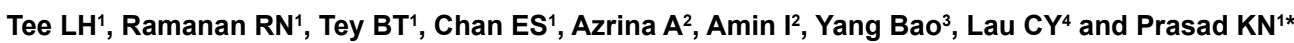

${ }^{1}$ School of Engineering, Monash University Malaysia, 47500, Malaysia

${ }^{2}$ Department of Nutrition and Dietetics, Faculty of Medicine and Health Sciences, Universiti Putra Malaysia, Selangor, Malaysia

${ }^{3}$ Key Laboratories of Plant Resources, Conservation and Sustainable Utilization, South China Botanical Garden, Chinese Academy of Sciences, Guangzhou, China

${ }^{4}$ Fruits and Post-Harvest Technology Section, Semongok Agriculture Research Centre, Department of Agriculture, Sarawak, Malaysia

\begin{abstract}
Nowadays, people are gaining interest on natural products due to the health conscious and awareness of the side effect caused by synthetic products. Malaysia is one of the top biodiversity, which host rich diversity of indigenous fruit, which could be a potential source of nutraceutical, antioxidant and pharmaceuticals. Dacryodes rostrata is an indigenous fruit found in Sarawak, Malaysia and is reported to be rich in oil, protein, minerals and could be a potential source of natural antioxidants. Thus, in this work, antioxidant components and antioxidant capacities of the extracts of peel, pulp and seeds of $D$. rostrata were evaluated. Total phenolic (1008 $\pm 24 \mathrm{mg} / \mathrm{g}$ GAE DW) and flavonoid contents $(2551 \pm 37 \mathrm{mg} / \mathrm{g}$ QE DW) of the seed extracts were higher when compared to peel and the pulp extracts. In addition, 1,1-diphenyl-2-picryl hydrazyl (DPPH) free radical scavenging activity of the seed extracts were higher (63\%), compared to peel $(25 \%)$ and pulp (18\%). Ferric reducing antioxidant power (FRAP) values of the seeds $(1.25 \mathrm{mM}$ $\mathrm{FeSO}_{4}$ equivalent) exhibited highest activity when, compared with peel $(0.29 \mathrm{mM} \mathrm{FeSO}$ equivalent $)$ and pulp $(0.90 \mathrm{mM}$ $\mathrm{FeSO}_{4}$ equivalent). Seed extract also showed highest total antioxidant capacity determined by phosphomolybdenum method. Liquid chromatography-mass spectra (LC-MS) of the seed extracts from $D$. rostrata revealed the presence of phytochemicals in the form of gallic acid, ellagic acid, catechin, epicatechin and apigenin. Thus, D.rostrata seed extracts is having potent antioxidant capacity and could be used as a natural source of antioxidants.
\end{abstract}

Keywords: Antioxidant; Dacryodes rostrata; Extraction; Flavonoids; Phenolic compounds

\section{Introduction}

Since ancient time, human have relied on mother nature for the basic needs including food, shelters, flavors, fragrances and also medicine [1]. Nowadays, people are getting more interested on the natural product remedies as they are more health conscious and aware of the side effects caused by synthetic product and drugs. Extensive studies and researches have been carried out on natural antioxidant and bioactive compounds in fruits, plants, seeds worldwide, due to the rising interest and market on the natural remedies and health supplement products [2].

Antioxidants were proven experimentally to be effective in lowering the oxidative stress and prevent the cellular damage caused by the free radicals generated in human body. Antioxidants have the ability to prevent and delay the onset of chronic diseases such as cardiovascular diseases, cancer, diabetes, stroke and dementia, which are caused by the oxidative stress $[3,4]$. However, the common synthetic antioxidants like butylatedhydroxytoulene (BHT), butylatedhydroxianisole (BHA) and tertiarybutylhydroquinone (TBHQ) have been reported to be toxic and carcinogenic to human body. This further lead to the exploration of natural, effective and economical antioxidant from natural sources such as fruits, vegetables and underutilized foods.

Malaysia is one of the top biodiversity spot in the world and has a rich diversity of indigenous fruits, that grow wildly in the peninsular Malaysia, Sabah and Sarawak. These indigenous fruits are the potential sources for the production of neutraceuticals, flavors and pharmaceuticals. However, these indigenous fruits are often underutilized due to their unknown features, and economical potential which have not been fully explored [4]. Several underutilized fruits are reported to be rich in bioactive compounds with prominent antioxidant capacity. For example, the oil obtained from the pulp and seed of an indigenous fruit, Canarium odontophyllum has shown to reduce oxidative stress and cholesterol level in animal models [5]. In addition, this fruit is also rich in phytochemicals like polyphenols which contribute to its antioxidant activity [6].
Dacryodes rostrata is one of the indigenous and underutilized fruit found in Sabah and Sarawak of Malaysia. The local communities commonly named it as kembayau. The fruit is ovoid to oblong in shape, dark purple in colour with a single seed in the center and is rich in proteins, minerals and oil. This fruit has been reported to possess significant nutritional content and rich in antioxidants in term of total phenolic and total flavonoid content [7]. This indicates that the extract from the $D$. rostrata has significant potential in developing into new herbal product or natural antioxidants. However, till date, this fruit is still underutilized and probably due to lacking of the information on its antioxidant activity as well as the phytochemicals that present in the fruit itself. Phytochemicals are bioactive compounds with antioxidant activity possessing various health benefits.

In this study, antioxidant activities of the $D$. rostrata fruits were evaluated. Different parts of the D. rostrata fruits, namely, peels, pulp and seeds were chosen in attempt to make a systematic comparisons among their antioxidant capacities and to identify the fraction with high antioxidant activity. In addition to that, the phytochemicals, which contribute to the antioxidant activity, were also been identified.

\section{Materials and Methods}

\section{Chemicals and reagents}

Methanol, hexane, hydrochloric acid, sulphuric acid (all analytical grades), acetonitrile and formic acid (HPLC grades) were purchased

${ }^{*}$ Corresponding author: Prasad KN, School of Engineering, Monash University Malaysia, 47500, Malaysia, Tel: +603 55146000 Ext 61242; Fax: +603 55146207; E-mail: nagendra.prasad@monash.edu

Received December 04, 2014; Accepted January 05, 2015; Published January 07, 2015

Citation: Tee LH, Ramanan RN, Tey BT, Chan ES, Azrina A, et al. (2015) Phytochemicals and Antioxidant Capacities from Dacryodesrostrata Fruits. Med chem 5: 023-027. doi:10.4172/2161-0444.1000238

Copyright: ( 2015 Tee LH, et al. This is an open-access article distributed under the terms of the Creative Commons Attribution License, which permits unrestricted use, distribution, and reproduction in any medium, provided the original author and source are credited. 
from Merch (Darmstadt, Germany) and ethanol (analytical grade) was obtained from Friendemann Schmidt (Parkwood, Australia). Gallic acid, sodium carbonate, Folin-Ciocalteu reagent, sodium nitrite, aluminum chloride, sodium hydroxide, quercetin, sodium phosphate, ammonium molybdate, Butylated hydroxytoluene (BHT), acetate buffer, 2,4,6-tripyridyl-s-triazine (TPTZ), ferric chloride, ferrous sulphate, 1,1-diphenyl-2-picryl hydrazyl (DPPH) were purchased from Sigma (St. Louis, USA).

\section{Sample preparation}

Fresh fruit of $D$. rostrata at mature stage was supplied by Agricultural Research Centre, Department of Agriculture, Sarawak, Malaysia. The fruits were transported to Monash University Malaysia in an icebox through airmail. The fruits without any physical damage with uniform shape and color were chosen and washed with tapped water and air-dried. The peel, pulp and seeds of the fruit were separated manually and freeze-dried. The freeze-dried samples were pulverized, sieved through $0.25 \mathrm{~mm}$ sieve and stored at $-20^{\circ} \mathrm{C}$ until further use.

\section{Ultrasonic extraction of sample for antioxidant assessment}

Ultrasonic extraction was carried out in accordance to the method by Khoo et al. [6]. Before the extraction, the lyophilized sample was defatted using hexane and the sample was subjected for low temperature oven drying overnight to remove the solvent. The defatted sample (10g) was extracted with $500 \mathrm{ml}$ of $50 \%$ ethanol with solid: solvent ratio of (1:50) using an ultrasonic cleaner (Thermoline, Thermo-6D, $40 \mathrm{kHz}$ ) at $30^{\circ} \mathrm{C}$ for 30 minutes. The solution was then filtered using vacuum filter and the filtrate was subjected to freeze drying to remove the solvent. The freeze-dried extract was then stored at $4^{\circ} \mathrm{C}$ for further analysis.

\section{Determination of total phenolic content (TPC)}

The total phenolic content was determined according to the method of Singleton and Rossi [8] and expressed as milligram gallic acid equivalent (GAE)/ 100 gram freeze-dried sample (FDS). A 100 $\mu \mathrm{L}$ aliquot of sample was added to $2 \mathrm{ml}$ of $20 \mathrm{~g} / \mathrm{L}$ sodium carbonate solution and incubated for 2 minutes. A $100 \mu \mathrm{L}$ of $50 \%$ Folin-Ciocalteu reagent was then added and the solution was allowed to stand for 90 minutes at $25^{\circ} \mathrm{C}$. The absorbance was measured at $750 \mathrm{~nm}$ against blank using a spectrophotometer (Genesys 10S UV-Vis, Thermo Scientific). The blank consisted of all the reagents and solvents without the sample. A standard calibration curve of gallic acid with concentration ranging from $0.5-1000 \mathrm{mg} / \mathrm{ml}$ was plotted and the total phenolic content was determined using the standard gallic acid calibration curve.

\section{Determination of total flavonoid content (TFC)}

Total flavonoid content was estimated following the aluminum chloride assay by Liu et al. [9]. In brief, $2 \mathrm{~mL}$ aliquot of sample was mixed with $0.2 \mathrm{~mL}$ of $5 \%$ sodium nitrite. After 5 minutes, $0.2 \mathrm{~mL}$ of $10 \%$ aluminum chloride was added to the mixture and mixed thoroughly. After 6 minutes, $2 \mathrm{~mL}$ of $1 \mathrm{M}$ sodium hydroxide was added and the mixture was made up to $5 \mathrm{~mL}$ using $50 \%$ ethanol and mixed thoroughly. The absorbance of mixture was read at $510 \mathrm{~nm}$ against blank using spectrophotometer. 50\% ethanol was used as blank. A standard calibration curve of $0.5-1000 \mathrm{mg} / \mathrm{ml}$ quercetin was plotted and the total flavonoid content was calculated using the standard calibration curve and expressed as milligram quercetin equivalent (QE)/ gram freeze-dried sample (FDS).

\section{Determination of antioxidant capacity by phosphomolybdenum method}

The antioxidant capacity was measured by the method of Prieto et al. [10]. Briefly, $0.1 \mathrm{~mL}$ of the sample was mixed with $1 \mathrm{~mL}$ of the reagent solution. The reagent which consisted of $0.6 \mathrm{M}$ sulphuric acid, $28 \mathrm{mM}$ sodium phosphate and $4 \mathrm{mM}$ ammonium molybdate. The mixture solution was then covered and incubated at $95{ }^{\circ} \mathrm{C}$ for 90 minutes. A blank sample containing all reagent and solvent without the sample was incubated under the same conditions. After the mixture was cooled down to room temperature, the absorbance was measured at $695 \mathrm{~nm}$ against the blank prepared earlier. A standard calibration curve of BHT was plotted to calculate the result and the antioxidant capacity was expressed as milligram BHT equivalent / gram freezedried sample (FDS).

\section{Determination of ferric reducing antioxidant power (FRAP)}

The ferric reducing antioxidant power was determined in accordance to the method by Kong et al. [7]. Three reagents including $300 \mathrm{mM}$ acetate buffer, $\mathrm{pH} 3.6,10 \mathrm{mM}$ TPTZ in $40 \mathrm{mM} \mathrm{HCl}$ and 20 $\mathrm{mM} \mathrm{FeCl}_{3}$ were prepared. The FRAP reagent was prepared by mixing acetate buffer, TPTZ solution and $\mathrm{FeCl}_{3}$ solution in proportion of 10:1:1 (v/v/v). An aliquot $(50 \mu \mathrm{L})$ of the sample was mixed with 3 $\mathrm{mL}$ of the FRAP reagent and incubated at $37^{\circ} \mathrm{C}$ for 30 minutes. The absorbance was measured at $593 \mathrm{~nm}$ against distilled water as blank. Ferrous sulphate $(0-1000 \mu \mathrm{M})$ was used to construct calibration curve and the results were expressed as $\mathrm{mmol} \mathrm{Fe}^{2+} / 100$ gram freeze-dried sample (FDS).

\section{Determination of 1,1-diphenyl-2-picryl hydrazyl (DPPH) free radicals scavenging activities}

The method of Prasad et al. [11] was adapted to determine the DPPH free radical scavenging activities of the extract. $0.1 \mathrm{~mL}$ sample was added into $0.9 \mathrm{~mL}$ of $200 \mu \mathrm{M}$ DPPH solution dissolved in methanol and mixed thoroughly. The mixture was then incubated for 30 minutes at $28^{\circ} \mathrm{C}$ in dark and the absorbance was measured at $515 \mathrm{~nm}$ against blank. Methanol was used as blank and the control contained all the reagents without the samples. The antioxidant activity was expressed as percentage of scavenging activity of DPPH radicals and calculated using the following formula:

\section{DPPH free radical scavenging activity, $\%=\left(A_{0}-A_{c}\right) / A_{0} \mathrm{x} \%$}

Where $\mathrm{A}_{0}$ is the absorbance of control, $\mathrm{A}_{\mathrm{c}}$ is the absorbance of the sample.

\section{LC-MS analysis}

The chromatography was performed on an Agilent 1290 Infinity LC system coupled to Agilent 6520 Accurate-Mass Q-TOF mass spectrometer with dual ESI source using an Agilent Zorbax SB-C18 column $(2.1 \times 150 \mathrm{~mm}, 3.5 \mu \mathrm{m})$. Flow rate was $0.4 \mathrm{~mL} / \mathrm{min}$ and the injection volume was $1 \mu \mathrm{L}$. Mobile phases consisted of $0.1 \%$ formic acid is Milli-Q water (A) and $100 \%$ Acetonitrile with $0.1 \%$ formic acid (B). The column was equilibrated for $5 \mathrm{~min}$ prior to each analysis. Separation was carried in 20 min under the following conditions: $0 \mathrm{~min}, 5 \% \mathrm{~B} ; 16$ min, $40 \%$ B; 20 min, 95\% B, $20.1 \mathrm{~min}, 100 \%$ B. The MS acquisition was performed in negative ionization information acquisition (IDA) between $\mathrm{m} / \mathrm{z} 115-1100$. The retention time and the mass obtained for the components were compared to the built in Metabolomics database.

\section{Results and Discussions}

\section{Antioxidant capacities}

From the experiment, the extract from kembayau seed exhibited a higher amount of both the TPC and TFC content (1007.96 mg GAE/g $\mathrm{dw}$ and $2550.90 \mathrm{mg} \mathrm{QE} / \mathrm{g} \mathrm{dw}$ respectively) followed by peel (560.28 $\mathrm{mg} \mathrm{GAE} / \mathrm{g} \mathrm{dw}$ and $556.92 \mathrm{mg} \mathrm{QE} / \mathrm{g} \mathrm{dw}$ ) and lastly pulp (273.03 mg 
GAE/g dw and 287.24 mg QE/g dw respectively). The results were in accordance to the study done on kembayau and other underutilized fruits where the seed contained higher amount of phenolic and flavonoid followed by peel and pulp $[7,12]$.

The antioxidant assays results were shown in Figures 1-3. From the DPPH radical scavenging activities result, the extract from seed exhibit highest scavenging activity and had superior antioxidant activity over the commercial synthetic antioxidant, BHT. Similar trends were observed in both FRAP and phosphomolybdenum analysis where the extract from seed exhibit the highest antioxidant capacity and is superior over both the BHT and ascorbic acid; the commercial antioxidants. The trends were in agreement with the study done by Kong et al. [7] and Guo et al. [13], who reported that the antioxidant capacities were higher in seed followed by peel and pulp for kembayau and other fruits.

\section{Phytochemicals}

Phytochemicals are known to play an important role in prevention of many major diseases. Since, D. rostrata seeds exhibited high TPC, TFC and antioxidant activity, it was subjected to phytochemical analysis. From the LC-MS chromatogram of $D$. rostrata seeds, 9 polyphenols were tentatively identified (Table 1). Among them,

\section{DPPH scavenging activity of different parts of kembayau}

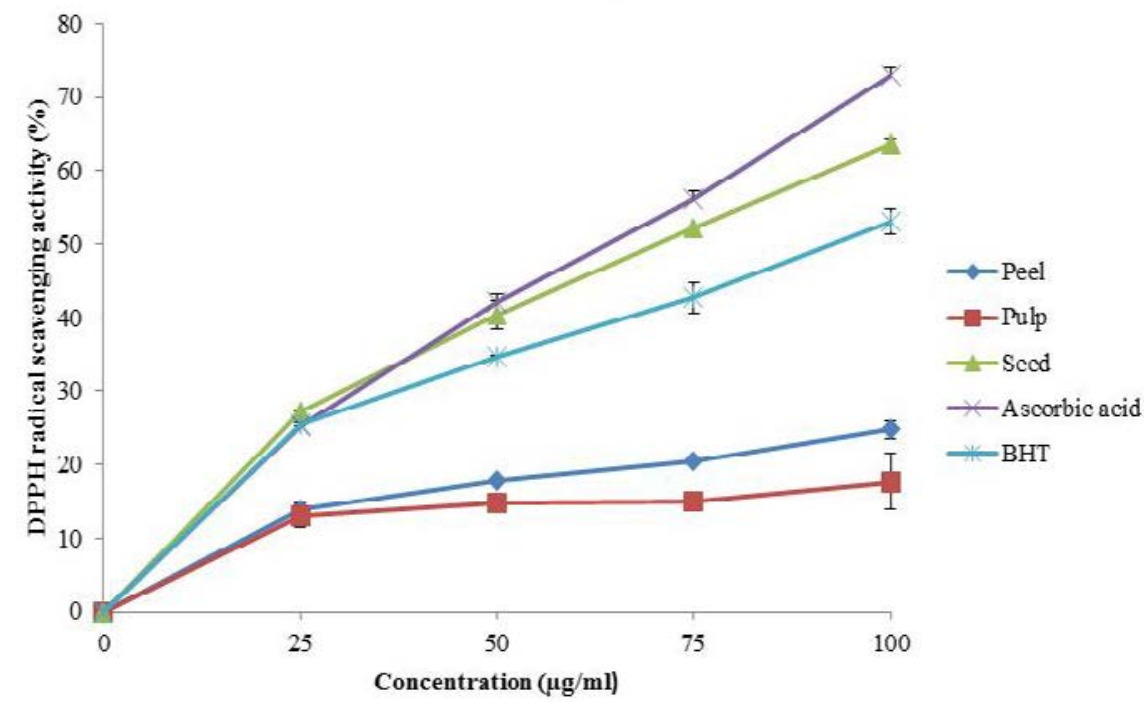

Figure 1: DPPH radical scavenging activities for different parts of kembayau, ascorbic acid and BHT.

\section{FRAP analysis of different parts of kembayau} fruit

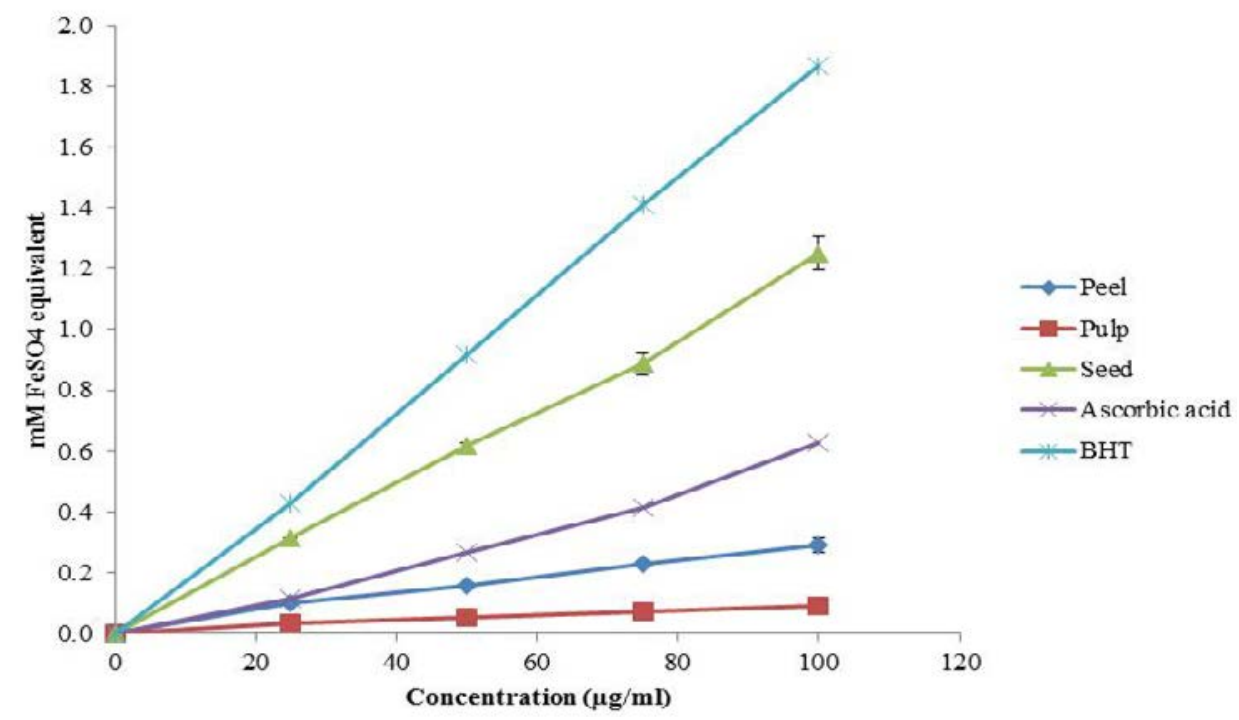

Figure 2: Ferric reducing antioxidant powers for different parts of kembayau, ascorbic acid and BHT. 


\section{Phosphomolybdenum analysis of different parts of kembayau}

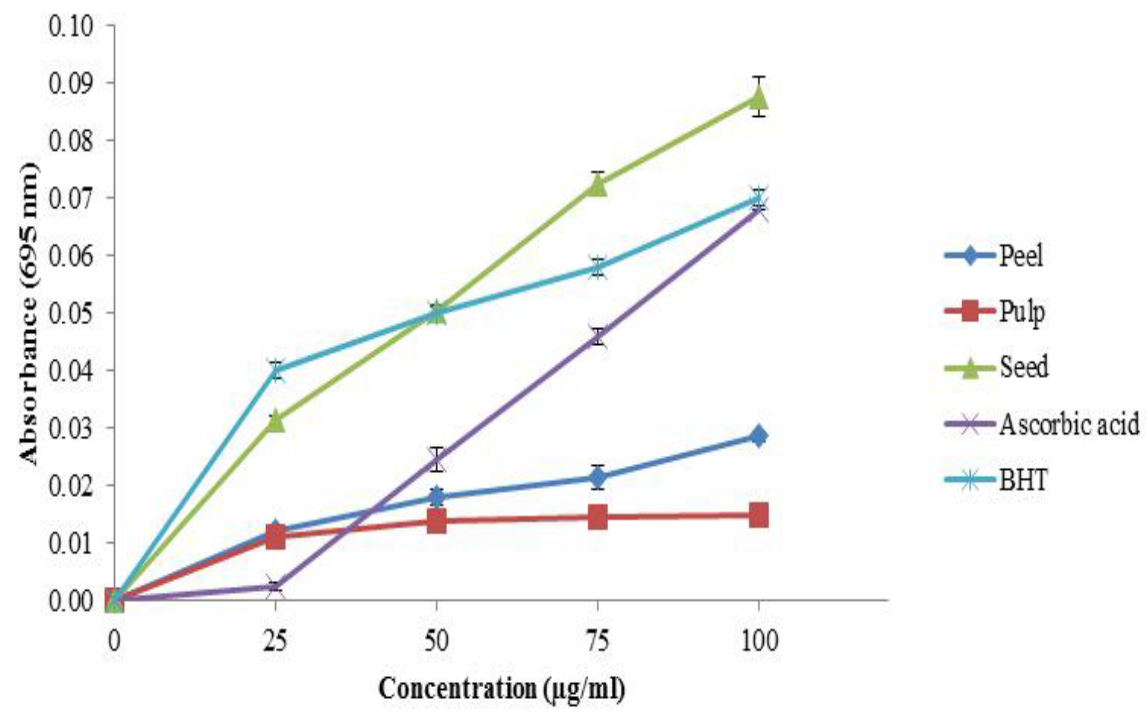

Figure 3: Antioxidant capacities by phosphomolybdenum method.

\begin{tabular}{|c|c|c|c|}
\hline No. & Compounds & $\begin{array}{c}\text { Retention time } \\
(\mathbf{m i n})\end{array}$ & $\mathbf{m} / \mathbf{z}$ \\
\hline $\mathbf{1}$ & Gallic acid & 2.052 & 169.014 \\
\hline $\mathbf{2}$ & Epigallocatechin & 3.624 & 305.067 \\
\hline $\mathbf{3}$ & Chlorogenic acid & 5.260 & 353.089 \\
\hline $\mathbf{4}$ & Apigenin 7-(4"-Z-p-coumarylglucoside) & 577.136 \\
\hline $\mathbf{5}$ & 1-Caffeoyl-4-deoxyquinic acid & 5.020 & 337.093 \\
\hline $\mathbf{6}$ & 5-O-Caffeoylshikimic acid & 5.295 & 335.078 \\
\hline $\mathbf{7}$ & (士)-Catechin & 5.682 & 289.073 \\
\hline $\mathbf{8}$ & Syringic acid & 5.682 & 197.046 \\
\hline $\mathbf{1 0}$ & Ellagic acid & 7.919 & 300.999 \\
\hline
\end{tabular}

Table 1: Phenolic compounds found in the seed of the kembayaufruitn and their chromatographic parameters.

chlorogenic acid and catechin were the major compounds along with minor compounds including gallic acid, ellagic acid among others. These phytochemicals are also reported from Dacryodes edulis. The presence of these phytochemcials confirmed the high antioxidant activity form $D$. rostrata seeds. The presence of these phytochemicals will be further verified by comparing the MS/MS fraction of the polyphenols standards.

\section{Conclusions}

In conclusion, the seed of kembayau contained highest amount of flavonoids and phenolic compound as well as exhibited highest antioxidant capacities. The phytochemicals present in the kembayau fruits were tentatively identified as chlorogenic acid, catechin, gallic acid, ellagic acid, epicatechin and apigenin. The presence of the phytochemicals and the antioxidant capacities confirmed that $D$. rostrata seeds could be used potentially as a readily accessible source of natural antioxidant or as a potential source for pharmaceutical supplements. Future works on the extraction of phytochemicals from the kembayau seed are worth investigating.

\section{Acknowledgement}

The financial support of e-Science Fund (02-02-10-SF0131) from Ministry of
Science, Technology \& Innovation (MOSTI), Malaysia to carry out the research work is thankfully acknowledged. The authors would also like to thank the use of laboratory facilities and the assistance of laboratory staffs from Chemical Engineering Discipline, School of Engineering, Monash University Malaysia.

\section{References}

1. Gurib-Fakim A (2006) Medicinal plants: traditions of yesterday and drugs of tomorrow. Mol Aspects Med 27: 1-93.

2. Chew LY, Prasad KN, Amin I, Azrina A, Lau CY (2011) Nutritional composition and antioxidant properties of Canarium odontophyllum Miq. (dabai) fruits. Journal of Food Composition and Analysis 24: 670-677.

3. Tabaraki R, Nateghi A (2011) Optimization of ultrasonic-assisted extraction of natural antioxidants from rice bran using response surface methodology. Ultrason Sonochem 18: 1279-1286.

4. Shakirin FH, Prasad KN, Ismail A, Yuon LC, Azlan A (2010) Antioxidant capacity of underutilized Malaysian Canariumodontophyllum (dabai) Miq. fruit. Journal of Food Composition and Analysis 23: 777-781.

5. Nurulhuda M, Azlan A, Ismail A, Amom Z, Shakirin FH (2012) Cholesterollowering and Artherosclerosis Inhibitory Effect of Sibu Olive in Cholesterol Fedrabbit. Asian Journal of Biochemistry 7 .

6. Khoo HE, Azlan A, Ismail A, Abas F (2012) Influence of different extraction media on phenolic contents and antioxidant capacity of defatted daba (Canariumodontophyllum) fruit. Food Analytical Methods 5: 339-350.

7. Kong KW, Chew LY, Prasad KN, Lau CY, Ismail A, et al. (2011) Nutritional 
Citation: Tee LH, Ramanan RN, Tey BT, Chan ES, Azrina A, et al. (2015) Phytochemicals and Antioxidant Capacities from Dacryodesrostrata Fruits. Med chem 5: 023-027. doi:10.4172/2161-0444.1000238

constituents and antioxidant properties of indigenous kembayau (Dacryodesrostrata (Blume) H. J. Lam) fruits. Food Research International 44: 2332-2338.

8. Liu H, Qiu N, Ding H, Yao R (2008) Polyphenols contents and antioxidant capacity of 68 Chinese herbals suitable for medical or food uses. Food Research International 41: 363-370.

9. Prieto P, Pineda M, Aguilar M (1999) Spectrophotometric quantitation of antioxidant capacity through the formation of a phosphomolybdenum complex: specific application to the determination of vitamin E. Anal Biochem 269: 337341.

10. Prasad KN, Yang B, Dong X, Jiang G, Zhang H, et al. (2009) Flavonoid contents and antioxidant activities from Cinnamomum species. Innovative Food Science and Emerging Technologies 10: 627-632.

11. Pande G, Akoh CC (2010) Organic acids, antioxidant capacity, phenolic content and lipid characterisation of Georgia-grown underutilized fruit crops. Food Chemistry 120: 1067-1075.

12. Guo C, Yang J, Wei J, Li Y, Xu J, et al. (2003) Antioxidant activities of peel, pulp and seed fractions of common fruits as determined by FRAP assay. Nutrition Research 23: 1719-1726.

13. Singleton V, Rossi JA (1965) Colorimetry of total phenolics with phosphomolybdic-phosphotungstic acid reagents. American journal of Enology and Viticulture 16: 144-158. 